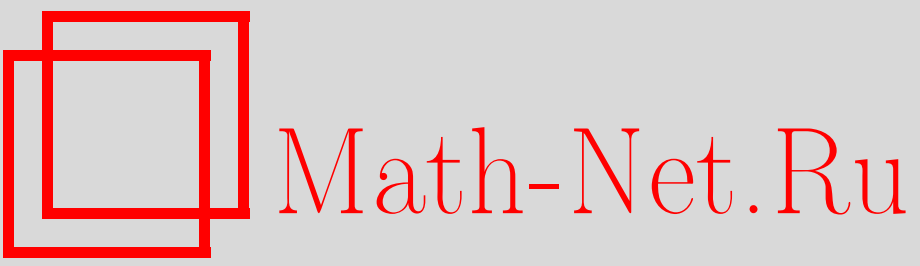

Г. Н. Губаль, М. А. Сташенко, Об улучшении оценки глобальной теоремы существования решения уравнений Боголюбова, ТМФ, 2005, том 145, номер 3, 420424

DOI: https://doi.org/10.4213/tmf1910

Использование Общероссийского математического портала Math-Net.Ru подразумевает, что вы прочитали и согласны с пользовательским соглашением http://www . mathnet.ru/rus/agreement

Параметры загрузки:

IP: 35.173 .137 .237

26 апреля 2023 г., 17:52:32 
ТЕОРЕТИЧЕСКАЯ

И МАТЕМАТИЧЕСКАЯ

ФИЗИКА

Toм 145, № 3

декабрь, 2005

(C) 2005 r.

Г.Н. Губаль*, М.А. Сташенко*

\section{ОБ УЛУЧШЕНИИ ОЦЕНКИ ГЛОБАЛЬНОЙ ТЕОРЕМЫ СУШЕСТВОВАНИЯ РЕШЕНИЯ УРАВНЕНИЙ БОГОЛЮБОВА}

Для одномерной системы частиц, взаимодействующих как упругие шары, улучшена оценка теоремы существования глобального решения задачи Коши для уравнений Боголюбова с начальными данными из пространства последовательностей измеримых функций.

Ключевые слова: уравнения Боголюбова, несимметричная система.

Глобальное решение начальной задачи для цепочки уравнений Боголюбова [1] с начальными данными из пространства последовательностей измеримых функций впервые построено в работах [2], [3]. В данной работе улучшена оценка теоремы сушествования глобального решения работы [2] для случая односортной системы.

1. Уравнения Боголюбова. Рассмотрим одномерную несимметричную систему тождественных частиц с массой $m=1$ и диаметром $\sigma>0$, которые взаимодействуют как упругие шары (стержни). Частицы в такой системе занимают только допустимые конфигурации $q_{i+1} \geqslant q_{i}+\sigma, i \in \mathbb{Z}^{1} \backslash\{0\}$. Конфигурации $W_{s}=\left\{\left(q_{-s_{2}}, \ldots, q_{s_{1}}\right) \in \mathbb{R}^{s_{1}+s_{2}} \mid\right.$ $q_{i+1}<q_{i}+\sigma$ хотя бы для одной пары $(i, i+1) \in\left\{\left(-s_{2},-s_{2}+1\right), \ldots,(-1,1), \ldots\right.$, $\left.\left.\left(s_{1}-1, s_{1}\right)\right\}\right\}$ называются запрешенными.

Состояние этой системы описывается последовательностью функций распределения $F(t)=\left\{F_{s}\left(t, x_{-s_{2}}, \ldots, x_{s_{1}}\right)\right\}_{s=s_{1}+s_{2} \geqslant 0}$, которые при $t \geqslant 0$ удовлетворяют уравнениям Боголюбова:

$$
\begin{aligned}
& \frac{\partial}{\partial t} F_{s}\left(t, x_{-s_{2}}, \ldots, x_{s_{1}}\right)=-(\mathcal{H} F(t))_{s}\left(x_{-s_{2}}, \ldots, x_{s_{1}}\right)+ \\
& \quad+\int_{0}^{\infty} d V V\left(F_{s+1}\left(t, x_{-s_{2}}, \ldots, x_{s_{1}-1} ; q_{s_{1}}, v_{s_{1}}^{*}\left(v_{s_{1}}, V\right) ; q_{s_{1}}+\sigma, v_{s_{1}+1}^{*}\left(v_{s_{1}}, V\right)\right)-\right.
\end{aligned}
$$

* Волынский государственный университет им. Леси Украинки, г. Луцк, Украина. E-mail: hhm@lt.ukrtel.net, smo@univer.lutsk.ua 


$$
\begin{aligned}
& \left.-F_{s+1}\left(t, x_{-s_{2}}, \ldots, x_{s_{1}} ; q_{s_{1}}+\sigma, v_{s_{1}}-V\right)\right)+ \\
& +\int_{0}^{\infty} d V V\left(F _ { s + 1 } \left(t, v_{-\left(s_{2}+1\right)}^{*}\left(v_{-s_{2}}, V\right), q_{-s_{2}}-\right.\right. \\
& \left.-\sigma ; v_{-s_{2}}^{*}\left(v_{-s_{2}}, V\right), q_{-s_{2}} ; x_{-\left(s_{2}-1\right)}, \ldots, x_{s_{1}}\right)- \\
& \left.-F_{s+1}\left(t, v_{-s_{2}}+V, q_{-s_{2}}-\sigma ; x_{-s_{2}}, \ldots, x_{s_{1}}\right)\right), \quad s=s_{1}+s_{2} \geqslant 1,
\end{aligned}
$$

где $\mathcal{H}$ - оператор Лиувилля:

$$
\begin{gathered}
(\mathcal{H} F(t))_{s}\left(x_{-s_{2}}, \ldots, x_{s_{1}}\right)=\sum_{i=-s_{2}}^{s_{1}} v_{i} \frac{\partial}{\partial q_{i}} F_{s}\left(t, x_{-s_{2}}, \ldots, x_{s_{1}}\right), \\
v_{i}^{*}\left(v_{i}, V\right)=v_{i}+V, \quad v_{i+1}^{*}\left(v_{i}, V\right)=v_{i} .
\end{gathered}
$$

2. Глобальное решение задачи Коши для уравнений Боголюбова. Решение задачи Коши для уравнений (1) в виде ряда итераций [2] имеет вид

$$
\begin{aligned}
F(t)= & U(t) F^{0}=\sum_{n=0}^{\infty} \int_{0}^{t} d t_{1} \cdots \int_{0}^{t_{n-1}} d t_{n} S\left(t_{1}-t\right)\left[\mathcal{H}, \mathfrak{a}^{+}+\mathfrak{a}^{-}\right] S\left(t_{2}-t_{1}\right) \times \cdots \\
& \cdots \times S\left(t_{n}-t_{n-1}\right)\left[\mathcal{H}, \mathfrak{a}^{+}+\mathfrak{a}^{-}\right] S\left(-t_{n}\right) F^{0}
\end{aligned}
$$

где $S(t)=\bigoplus_{s=0}^{\infty} S^{s}(t)$ :

$$
\begin{aligned}
& \left(S^{s}(t) f_{s}\right)\left(x_{-s_{2}}, \ldots, x_{s_{1}}\right)= \\
& \quad=\left\{\begin{array}{lll}
f_{s}\left(X_{-s_{2}}(t), \ldots, X_{s_{1}}(t)\right), & \text { если } & \left(x_{-s_{2}}, \ldots, x_{s_{1}}\right) \in \mathbb{R}^{s} \times\left(\mathbb{R}^{s} \backslash W_{s}\right), \\
0, & \text { если } & \left(q_{-s_{2}}, \ldots, q_{s_{1}}\right) \in W_{s},
\end{array}\right.
\end{aligned}
$$

$X_{i}(t) \equiv X_{i}\left(t, x_{-s_{2}}, \ldots, x_{s_{1}}\right), i=-s_{2}, \ldots, s_{1},-$ решение начальной задачи для уравнений Гамильтона системы $s$ частищ с начальными данными $X_{i}\left(0, x_{-s_{2}}, \ldots, x_{s_{1}}\right)=x_{i}, i=$ $-s_{2}, \ldots, s_{1}$,

$$
\begin{gathered}
\left(\mathfrak{a}^{+} f\right)_{s}\left(x_{-s_{2}}, \ldots, x_{s_{1}}\right)=\int d x_{s_{1}+1} f_{s_{1}+1+s_{2}}\left(x_{-s_{2}}, \ldots, x_{s_{1}+1}\right), \\
\left(\mathfrak{a}^{-} f\right)_{s}\left(x_{-s_{2}}, \ldots, x_{s_{1}}\right)=\int d x_{-\left(s_{2}+1\right)} f_{s_{1}+s_{2}+1}\left(x_{-\left(s_{2}+1\right)}, \ldots, x_{s_{1}}\right), \\
\left(\left[\mathcal{H}, \mathfrak{a}^{+}+\mathfrak{a}^{-}\right] f\right)_{s}\left(x_{-s_{2}}, \ldots, x_{s_{1}}\right)= \\
=\int_{\mathbb{R}^{1}} d v_{s_{1}+1}\left(v_{s_{1}+1}-v_{s_{1}}\right) f_{s+1}\left(x_{-s_{2}}, \ldots, x_{s_{1}} ; q_{s_{1}}+\sigma, v_{s_{1}+1}\right)+ \\
+\int_{\mathbb{R}^{1}} d v_{-\left(s_{2}+1\right)}\left(v_{-s_{2}}-v_{-\left(s_{2}+1\right)}\right) f_{s+1}\left(v_{-\left(s_{2}+1\right)}, q_{-s_{2}}-\sigma ; x_{-s_{2}}, \ldots, x_{s_{1}}\right) .
\end{gathered}
$$

Рассмотрим начальные данные $F^{0}$ из пространства $L_{\xi, \beta}^{\infty}$ двойных последовательностей $f=\left\{f_{s}\left(x_{-s_{2}}, \ldots, x_{s_{1}}\right)\right\}_{s=s_{1}+s_{2} \geqslant 0}$ измеримых функций $f_{s}\left(x_{-s_{2}}, \ldots, x_{s_{1}}\right)$, равных нулю на множестве $W_{s}$ с нормой

$$
\|f\|=\sup _{s \geqslant 0} \xi^{-s} \sup _{\substack{s_{1}, s_{2} \geqslant 0 \\ s_{1}+s_{2}=s}} \sup _{x_{-s_{2}}, \ldots, x_{s_{1}}}\left|f_{s}\left(x_{-s_{2}}, \ldots, x_{s_{1}}\right)\right| \exp \left(\beta \sum_{i=-s_{2}}^{s_{1}} \frac{v_{i}^{2}}{2}\right),
$$


где $\xi, \beta>0$ - числа.

Запишем формулу (2) покомпонентно. Для этого введем следуюшие операторы:

$$
\begin{gathered}
{\left[\mathcal{H}, \mathfrak{a}^{+}+\mathfrak{a}^{-}\right]=\stackrel{*}{\mathfrak{A}}(0)-\mathfrak{A}(0),} \\
\stackrel{*}{\mathfrak{A}}(0)=\stackrel{*}{\mathfrak{A}}^{+}(0)+\stackrel{*}{\mathfrak{A}}^{-}(0), \quad \mathfrak{A}(0)=\mathfrak{A}^{+}(0)+\mathfrak{A}^{-}(0),
\end{gathered}
$$

где

$$
\begin{aligned}
&\left(\mathfrak{A}^{+}(0) f\right)_{s}\left(x_{-s_{2}}, \ldots, x_{s_{1}}\right)= \\
&=\int_{0}^{\infty} d V V f_{s+1}\left(x_{-s_{2}}, \ldots, x_{s_{1}-1} ; q_{s_{1}}, v_{s_{1}}^{*}\left(v_{s_{1}}, V\right) ; q_{s_{1}}+\sigma, v_{s_{1}+1}^{*}\left(v_{s_{1}}, V\right)\right), \\
&\left(\mathfrak{A}^{+}(0) f\right)_{s}\left(x_{-s_{2}}, \ldots, x_{s_{1}}\right)= \int_{0}^{\infty} d V V f_{s+1}\left(x_{-s_{2}}, \ldots, x_{s_{1}} ; q_{s_{1}}+\sigma, v_{s_{1}}-V\right), \\
&\left(\mathfrak{A}^{-}(0) f\right)_{s}\left(x_{-s_{2}}, \ldots, x_{s_{1}}\right)= \int_{0}^{\infty} d V V f_{s+1}\left(v_{-\left(s_{2}+1\right)}^{*}\left(v_{-s_{2}}, V\right), q_{-s_{2}}-\right. \\
&\left.-\sigma ; v_{-s_{2}}^{*}\left(v_{-s_{2}}, V\right), q_{-s_{2}} ; x_{-\left(s_{2}-1\right)}, \ldots, x_{s_{1}}\right), \\
&\left(\mathfrak{A}^{-}(0) f\right)_{s}\left(x_{-s_{2}}, \ldots, x_{s_{1}}\right)= \int_{0}^{\infty} d V V f_{s+1}\left(v_{-s_{2}}+V, q_{-s_{2}}-\sigma ; x_{-s_{2}}, \ldots, x_{s_{1}}\right) .
\end{aligned}
$$

Определим также

$$
\mathfrak{A}^{*}\left(\tau_{i}\right)=S\left(\tau_{i}\right) \mathfrak{A}^{*}(0), \quad \mathfrak{A}^{ \pm}\left(\tau_{i}\right)=S\left(\tau_{i}\right) \mathfrak{A}^{ \pm}(0),
$$

где $\tau_{i}=t_{i}-t_{i-1}$. Таким образом, выражение для ряда итераций $(2)$ примет вид

$$
\begin{aligned}
F_{s}\left(t, x_{-} s_{2}\right. & \left.\ldots, x_{s_{1}}\right)=\sum_{n=0}^{\infty} \int_{0}^{t} d t_{1} \ldots \int_{0}^{t_{n-1}} d t_{n} \sum_{k=0}^{n} \sum_{1 \leqslant j_{1}<\cdots<j_{k}}^{n}(-1)^{n-k} \times \\
& \times\left(\mathfrak{A}\left(\tau_{1}\right) \ldots \mathfrak{A}\left(\tau_{j_{1}-1}\right) \mathfrak{A}\left(\tau_{j_{1}}\right) \mathfrak{A}\left(\tau_{j_{1}+1}\right) \ldots \mathfrak{A}\left(\tau_{j_{k}-1}\right) \times\right. \\
& \left.\times \stackrel{*}{\mathfrak{A}}\left(\tau_{j_{k}}\right) \mathfrak{A}\left(\tau_{j_{k}+1}\right) \ldots \mathfrak{A}\left(\tau_{n}\right) S\left(-t_{n}\right) F^{0}\right)_{s}\left(x_{-s_{2}}, \ldots, x_{s_{1}}\right) .
\end{aligned}
$$

Определим эволюционный оператор (2) в $L_{\xi, \beta}^{\infty}$. Справедлива следующая

Tеорема. Если $F^{0} \in L_{\xi, \beta}^{\infty}$, то последовательность $F(t)(2)$ существует, для функиий $F_{s}\left(t, x_{-s_{2}}, \ldots, x_{s_{1}}\right)$ сходится равномерно по $\left(x_{-s_{2}}, \ldots, x_{s_{1}}\right)$ на каждом компакте при $t \in(-\infty,+\infty)$ и справедлива оченка

$$
\left|F_{s}\left(t, x_{-s_{2}}, \ldots, x_{s_{1}}\right)\right| \leqslant\left\|F^{0}\right\| \xi^{s} \exp \left(-\frac{\beta}{3} \sum_{i=-s_{2}}^{s_{1}} \frac{v_{i}^{2}}{2}\right) e^{t /\left(t_{0} \sqrt{e}\right)}
$$

где $t_{0} \equiv(1 / 3) \beta\left(2^{3} \sqrt{2 \pi} \xi\right)^{-1}$. Функиии $F_{s}(t)$ являются слабыми решениями уравнений Боголюбова (1). 
ДокАЗАТЕЛЬСТВо. Построим оценку типичного слагаемого $n$-го члена ряда (4), например

$$
\left(\mathfrak{A}^{+}\left(\tau_{1}\right) \ldots \mathfrak{A}^{+}\left(\tau_{n}\right) S\left(-t_{n}\right) F^{0}\right)_{s}\left(x_{-s_{2}}, \ldots, x_{s_{1}}\right) .
$$

Перейдем к новым переменным интегрирования $V_{i}=v_{s_{1}+i-1}-v_{s_{1}+i}$, где $i=1, \ldots, n$. Подынтегральная функция из выражения $\left(\mathfrak{A}^{+}\left(\tau_{1}\right) \ldots \mathfrak{A}^{+}\left(\tau_{n}\right) S\left(-t_{n}\right) F^{0}\right)_{s}\left(x_{-s_{2}}, \ldots, x_{s_{1}}\right)$ примет вид

$$
\prod_{i=1}^{n}\left(V_{s_{1}+i-1}\left(t_{i}-t_{i-1}, \ldots\right)-v_{s_{1}+i}\right) F_{s+n}^{0}\left(X_{-s_{2}}\left(-t_{n}, \ldots\right), \ldots, X_{s_{1}+n}\left(-t_{n}, \ldots\right)\right),
$$

где функции $V_{s_{1}+i-1}\left(t_{i}-t_{i-1}, \ldots\right), X_{j}\left(-t_{n}, \ldots\right)$ зависят от соответствующих начальных данных согласно формуле (4) и определениям (3).

Для произвольных $F^{0} \in L_{\xi, \beta}^{\infty}$, используя закон сохранения энергии, выражение (6) можно оценить согласно неравенству

$$
\left|F_{s+n}^{0}\left(X_{-s_{2}}\left(-t_{n}, \ldots\right), \ldots, X_{s_{1}+n}\left(-t_{n}, \ldots\right)\right)\right| \leqslant \xi^{n+s}\left\|F^{0}\right\| \exp \left(-\beta \sum_{i=-s_{2}}^{s_{1}+n} \frac{v_{i}^{2}}{2}\right)
$$

и следуюшей лемме.

Лемма. Справедливо неравенство

$$
\prod_{i=1}^{n}\left(\left|V_{s_{1}+i-1}\left(t_{i}-t_{i-1}, \ldots\right)\right|+\left|v_{s_{1}+i}\right|\right) \exp \left(-\alpha \sum_{j=-s_{2}}^{s_{1}+n} v_{j}^{2}\right) \leqslant\left(\frac{2}{\alpha}\right)^{n / 2} e^{-n / 2}
$$

Докажем лемму. Используя неравенство Коши и закон сохранения энергии, получаem

$$
\begin{aligned}
& \prod_{i=1}^{n}\left(\left|V_{s_{1}+i-1}\left(t_{i}-t_{i-1}, \ldots\right)\right|+\left|v_{s_{1}+i}\right|\right) \leqslant \\
& \leqslant\left(\frac{1}{n} \sum_{i=1}^{n}\left(\left|V_{s_{1}+i-1}\left(t_{i}-t_{i-1}, \ldots\right)\right|+\left|v_{s_{1}+i}\right|\right)\right)^{n} \leqslant \\
& \leqslant\left(\sqrt{\frac{1}{n} \sum_{i=1}^{n}\left(V_{s_{1}+i-1}\left(t_{i}-t_{i-1}, \ldots\right)\right)^{2}}+\sqrt{\frac{1}{n} \sum_{i=1}^{n} v_{s_{1}+i}^{2}}\right)^{n} \leqslant \\
& \leqslant\left(\sqrt{2\left(\frac{1}{n} \sum_{i=1}^{n}\left(V_{s_{1}+i-1}\left(t_{i}-t_{i-1}, \ldots\right)\right)^{2}+\frac{1}{n} \sum_{i=1}^{n} v_{s_{1}+i}^{2}\right)}\right)^{n} \leqslant \\
& \leqslant\left(\frac{4}{n}\right)^{n / 2}\left(\sum_{j=-s_{2}}^{s_{1}+n} v_{j}^{2}\right)^{n / 2} .
\end{aligned}
$$

Вычисляя значение

$$
\sup _{\left\{v_{j}\right\}}\left(\frac{4}{n}\right)^{n / 2}\left(\sum_{j=-s_{2}}^{s_{1}+n} v_{j}^{2}\right)^{n / 2} \exp \left(-\alpha \sum_{j=-s_{2}}^{s_{1}+n} v_{j}^{2}\right)=\left(\frac{2}{\alpha}\right)^{n / 2} e^{-n / 2},
$$


приходим к неравенству (7).

Аналогично оцениваются слагаемые, определяющиеся операторами $\mathfrak{A}^{+}\left(\tau_{i}\right), \mathfrak{A}^{+}\left(\tau_{i}\right)$ и $\mathfrak{A}^{-}\left(\tau_{i}\right), \stackrel{*}{\mathfrak{A}}\left(\tau_{i}\right)$ и $\mathfrak{A}\left(\tau_{i}\right)$.

Положим $\alpha=\beta / 6$, тогда каждый интеграл по скорости в (4) оценим величиной $\sqrt{\pi \alpha^{-1}}$. В итоге получаем оценку (5). Теорема доказана.

3. Замечание. Отметим, что полученные в работе [2] оценки имеют вид

$$
\begin{gathered}
\prod_{i=1}^{n}\left(\left|V_{s_{1}+i-1}\left(t_{i}-t_{i-1}, \ldots\right)\right|+\left|v_{s_{1}+i}\right|\right) \exp \left(-\alpha \sum_{j=-s_{2}}^{s_{1}+n} v_{j}^{2}\right) \leqslant\left(\frac{2}{\alpha}\right)^{n / 2} \sqrt{n !} \\
\left|F_{s}\left(t, x_{-s_{2}}, \ldots, x_{s_{1}}\right)\right| \leqslant\left\|F^{0}\right\| \xi^{s} \exp \left(-\frac{\beta}{3} \sum_{i=-s_{2}}^{s_{1}} \frac{v_{i}^{2}}{2}\right) \sum_{n=0}^{\infty} \frac{1}{\sqrt{n !}}\left(\frac{t}{t_{0}}\right)^{n}
\end{gathered}
$$

Из того, что

$$
\begin{gathered}
\left(\frac{2}{\alpha}\right)^{n / 2} e^{-n / 2}<\left(\frac{2}{\alpha}\right)^{n / 2} \sqrt{n !} \\
\left\|F^{0}\right\| \xi^{s} \exp \left(-\frac{\beta}{3} \sum_{i=-s_{2}}^{s_{1}} \frac{v_{i}^{2}}{2}\right) e^{t /\left(t_{0} \sqrt{e}\right)}<\left\|F^{0}\right\| \xi^{s} \exp \left(-\frac{\beta}{3} \sum_{i=-s_{2}}^{s_{1}} \frac{v_{i}^{2}}{2}\right) \sum_{n=0}^{\infty} \frac{1}{\sqrt{n !}}\left(\frac{t}{t_{0}}\right)^{n},
\end{gathered}
$$

получаем улучшение оценки теоремы сушествования глобальных решений, которые описывают эволюцию произвольных неравновесных состояний одномерной системы бесконечного числа частиц.

\section{Список литературы}

[1] Н. Н. Боголюбов. Проблемы динамической теории в статистической физике. М.: Гостехиздат, 1946.

[2] В.И. Герасименко. ТМФ. 1992. Т. 91. № 1. С. 120-128.

[3] C. Cercignani, V.I. Gerasimenko, D. Ya. Petrina. Many-Particle Dynamics and Kinetic Equations. Dordrecht: Kluwer, 1997.

Поступила в редакцию 8.II.2005 г., после доработки 23.III.2005 г. 